IP Periodica Polytechnica

Mechanical Engineering

57(2), pp. 23,27 2013

DOI: 10.3311/PPme.7041

Creative Commons Attribution (1)

RESEARCH ARTICLE

\section{An experimental and theoretical study on the effect of sampling time delay on the stability of a PI position controller of a hydraulic cylinder}

Csaba Hős, Balázs Kuti

Received 2013-04-15, accepted 2013-07-01

\begin{abstract}
The stability properties of the feedback control of a hydraulic cylinder is analysed with an emphasis on the effect of the control delay due to the discrete sampling. A simple theoretical model is developed for low-load cases, i.e. if the forces acting of the piston are small compared to the pressure forces inside the cylinder. It is shown that in the case of a traditional PI controller, there is an upper and lower limit on the proportional gain corresponding to significantly different mechanisms of stability loss. It is shown that the frequency of the appearing oscillations at low proportional gains is one range smaller than that the sampling frequency. The theoretical results are confirmed by measurements.
\end{abstract}

\section{Keywords}

Hydraulic cylinder $\cdot$ PI control $\cdot$ discrete-time system $\cdot$ position control $\cdot$ stability

\section{Acknowledgement}

This research was supported by grant OTKA PD 76478 and MTA's János Bolyai scholarship of C. Hö́s and has been developed in the framework of the project "Talent care and cultivation in the scientific workshops of BME" project. This project was supported by the grant TÁMOP-4.2.2.B-10/1-2010-0009.

\section{Csaba Hös}

Budapest University of Technology and Economics (BUTE) Dept. of Hydrodynamic Systems (HDS), H-1111, Budapest, Múegyetem rkp. 3, Hungary e-mail: csaba.hos@hds.bme.hu

\section{Balázs Kuti}

Budapest University of Technology and Economics (BUTE) Dept. of Hydrodynamic Systems (HDS), H-1111, Budapest, Múegyetem rkp. 3, Hungary

e-mail: balazsute@hotmail.com

\section{Introduction}

Hydraulic position servos are commonly used in industrial applications where large forces are to be exerted with high stiffness and fast response. Although these systems are strongly nonlinear, classic PI or PID technique is usually employed for feedback control, see [5] - especially in the industrial applications. The drawback of the linear approach is not only that important features are lost (dead zone, flow-through characteristics, etc.) but also that it becomes cumbersome to tune the control parameters in the presence of strong nonlinearities. To cope with these issues, several advanced control strategies were suggested, such as sliding mode control (see e.g. [7]), integrator backstepping (see e.g. [1]) or mixed fuzzy-PID/neural network compensating schemes (see [2]), just to mention a few.

This paper focuses on the issue of controller time delay, i.e. that the control output is computed with the help of the state variables measured in the past. The origin of the delay can be digital sampling (see e.g. [4] 8]), network control (as described in [3 10]) or the wave effects in the transmission lines (see [11]). Whichever type is present in the system, the stability of the controller is affected in an undesired way; i.e., the stable domain shrinks and oscillations emerge. The present study focuses on the dynamics of the actuator, unlike the majority of the corresponding literature, where the dynamics of the controlled object (usually represented by an equivalent spring-mass system) plays the central role.

We present the results of a series of measurements on the PI control of a hydraulic cylinder together with a simplified mathematical model that gives reasonably good agreement with the experiments, for low-load cases, i.e., if the forces acting on the controlled object are negligible compared to the force exerted by the piston. The rest of the paper is organized as follows. Section 2 describes the test rig and the implementation of the control law. Section 3 presents the mathematical model, which, in a nutshell, assumes linear relationship between the control signal and the velocity of the cylinder. The resulting linear map is then analysed with standard techniques and the stability boundaries are given. 


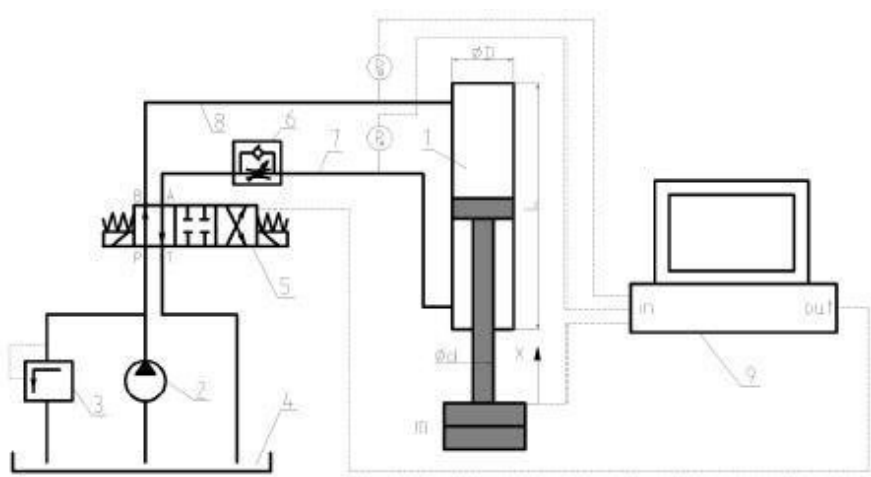

Fig. 1. Sketch of the text rig.

Section 3.3 presents the comparison of the experimental and theoretical results while Section 4 summarizes the work.

\section{Experimental set-up}

The sketch of the text rig is shown in Fig. 11 The hydraulic aggregate (2) (consisting of a positive displacement pump and a pressure relief valve) feeds the hydraulic cylinder (4) via a proportional valve (3). The displacement of the piston is measured by a capacitive displacement sensor. The signal of the displacement sensor is fed into a PC (5), which computes the output signal for the proportional valve. The tank (1) contains the hydraulic fluid and ensures its stable temperature.

The type of the cylinder is Bosch Rexroth CDM1Mp5 with diameters $D / d=50 / 28 \mathrm{~mm}$ and $150 \mathrm{~mm}$ stroke. The type of the proportional valve is Bosch Rexroth 4WRAE 10, whose input signal is $-10 \ldots 10 \mathrm{~V}$ with a dead zone (backlash) of width $-2 \ldots 2 \mathrm{~V}$. The internal diameter of the connecting hydraulic hoses is $12 \mathrm{~mm}$. The opening pressure of the relief valve was set to 100 bar. The material properties of the hydraulic oil are: $\rho=870 \mathrm{~kg} / \mathrm{m}^{3}, v=12.95 \mathrm{~mm}^{2} / \mathrm{s}$ and $E=1.9 \mathrm{GPa}$.

Classic PI control in a discrete form was implemented on a standard desktop PC under LabView environment. Let $\Delta t$ and $f$ denote the sampling time and frequency, respectively, with $\Delta t=1 / f$. The continuous form the the PI control is

$$
u(t)=-\left(P e(t)+I \int_{0}^{t} e(\tau) \mathrm{d} \tau\right),
$$

where $e(t)=x(t)-x_{d}(t)$ with $x_{d}(t)$ being the desired position. Due to the discrete sampling, we need the discrete version of (11). Let $t_{0}$ denote the initial time, $t_{i}$ the $i$ th sampling time, i.e. $t_{i}=t_{0}+i \Delta t$ and $t_{N}$ the last sampling. We have

$$
u\left(t_{N}+\Delta t\right):=u_{N+1}=-\left(P e_{N}+I C_{N}\right),
$$

with the update rule

$$
C_{N}=C_{N-1}+\left(e_{N-1}+e_{N}\right) \frac{\Delta t}{2},
$$

that is, the integral term is approximated with the help of the trapezoidal rule. Note that no differential term is used as it is rather cumbersome and inaccurate to differentiate a measured signal.

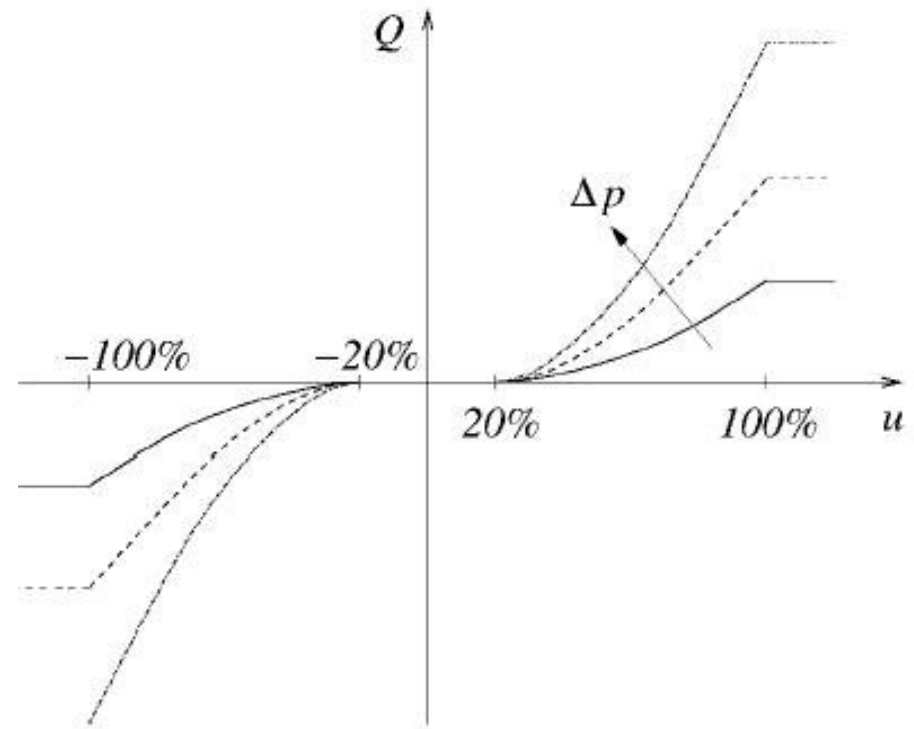

Fig. 2. Characteristic curve of a hydraulic proportional control valve.

Finally, the flow rate $Q$ is connected to the valve input signal $u$ via the characteristics of the control valve (element (3) in Fig. 11. This curve includes both dead zone and saturation parts as shown in Fig. 2 Typically, below $20 \%$ of the command signal, the flow rate is zero, which is followed by a slightly nonlinear curve up to the maximum value of the command signal, beyond which, as the flow-through area is constant, the flow rate remains constant for a given pressure difference.

Unless otherwise stated, we use the following parameter values: $u_{\max }=10 \mathrm{~V}, Q_{\max }=20 \mathrm{lit} / \mathrm{min}$ (maximum control signal and flow rate at nominal system pressure), $D=0.05, d=0.028$, $A_{D}=D^{2} \pi / 4, A_{d}=\left(D^{2}-d^{2}\right) \pi / 4$ and we use the 'mean' piston area $A_{p}=\left(A_{D}+A_{d}\right) / 2$. Typical system pressure was $\Delta p=50$ bars, which gives $F_{p}=A_{p} \Delta p=8.28 \mathrm{kN}$ piston force. The measurements were performed with a vertical cylinder which moved an object of $60 \mathrm{~kg}$ mass.

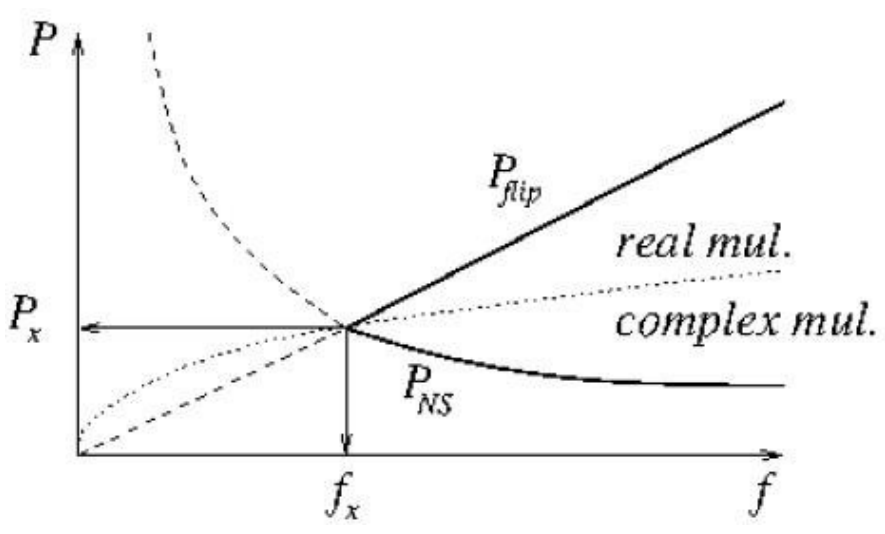

Fig. 3. Qualitative stability chart, the stable region is shaded.

\section{Theoretical study}

\subsection{Mathematical modelling}

In this section we develop a simplified mathematical model to capture the cylinder dynamics. We neglect the dynamics of the 
Fig. 4. Time histories started from the same initial condition $x_{0}=[0.1,0,0]$, with $P=0.6,1.5,9.0$, 10.1. The sampling frequency and the integrator gain are $f=10 \mathrm{~Hz}$ and $I=20$.
Fig. 5. Stable control regions for (a) $I=0$, (b) $I=5$, (c) $I=20$, (d) $I=50$. Solid line represent measurement, dashed line stands for theory. The circles denote the point where the simulations in Fig. 4 were performed.
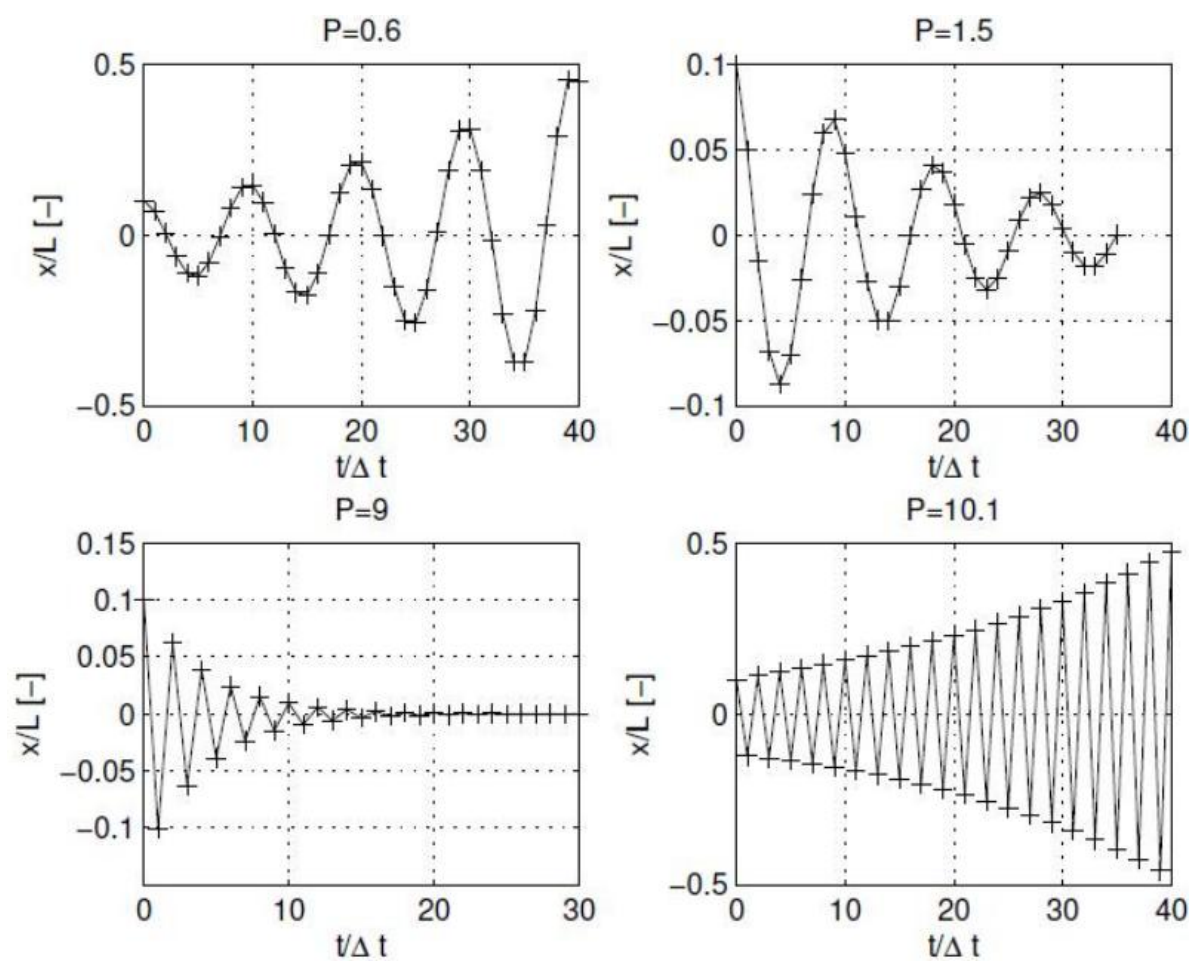

(a)

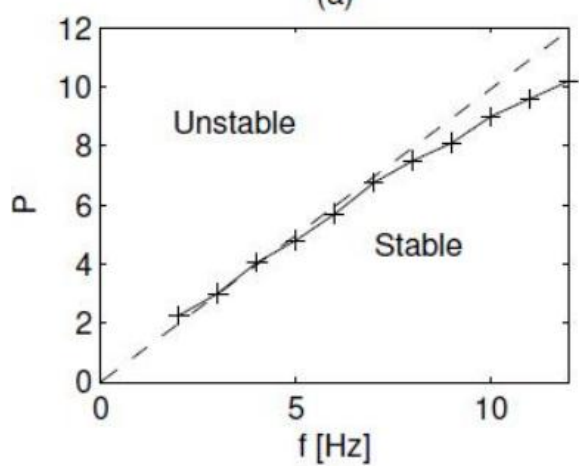

(c)

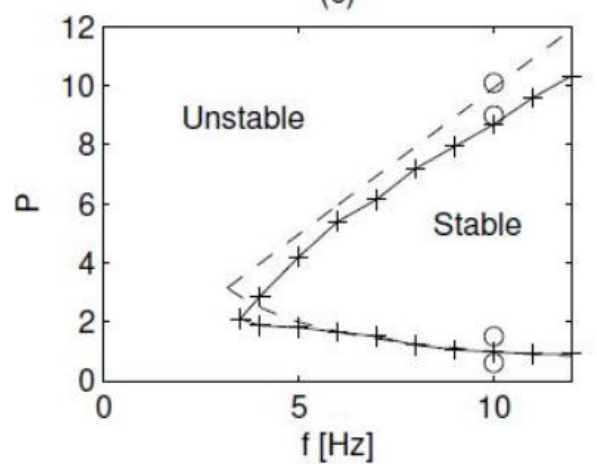

(b)

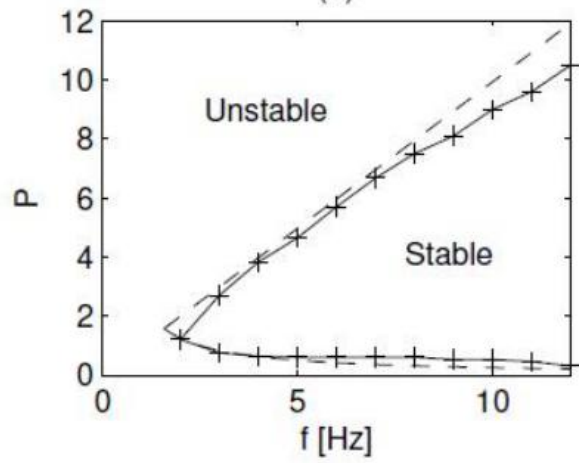

(d)

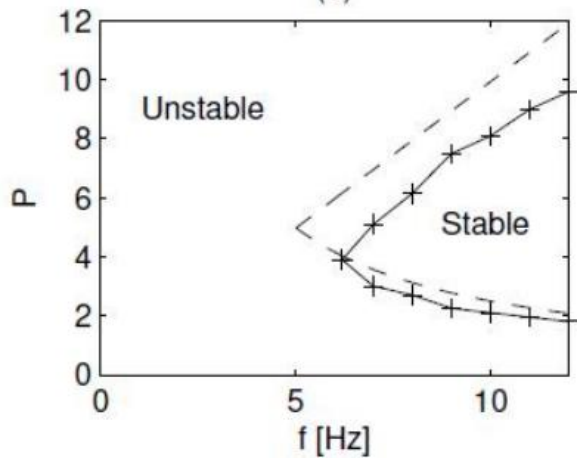

moved object as well as all the forces acting on it, notably friction and gravity. In other words, we consider cases with small, negligible load compared to the piston force, which is computed with the piston area and mean system pressure. Our first key assumption is that the internal pressure dynamics of the cylinder chambers can be neglected, hence the flow rate through the control valve and the velocity of the piston are coupled by the algebraic equation

$$
v_{\text {piston }}=\frac{Q}{A_{p}},
$$

which, due to the discrete sampling, gives the displacement update

$$
x_{N+1}=x_{N}+\frac{Q_{N}}{A_{p}} \Delta t .
$$

The flow rate $Q_{N}$ is a function of the valve input signal $u$ as already described in the previous section and is depicted in Fig. 2. However, in this study the second key assumption is that both the nonlinearity and the dead zone can be neglected and the valve characteristics can be replaced by a simple linear function: $Q=K u=Q_{\max } / u_{\max } u$. We also neglect the computation time 
needed to evaluate the control law, hence we have

$$
Q_{N}=K u_{N}=-K\left(P e_{N}+I C_{N}\right) \text {, }
$$

where $C_{N}$ is given by (3). Putting together (5), (6) and (3), we obtain

$$
\left(\begin{array}{c}
x_{N+1} \\
x_{N} \\
C_{N+1}
\end{array}\right)=\left(\begin{array}{ccc}
\alpha & \beta & \gamma \\
1 & 0 & 0 \\
\Delta t / 2 & \Delta t / 2 & 1
\end{array}\right)\left(\begin{array}{c}
x_{N} \\
x_{N-1} \\
C_{N}
\end{array}\right)
$$

with

$$
\beta=-I \frac{K \Delta t^{2}}{2 A_{p}}, \quad \alpha=1-P \frac{K \Delta t}{A_{p}}-\beta \quad \text { and } \quad \gamma=\beta \frac{2}{\Delta t},
$$

where $x_{d}=0$ was chosen.

\subsection{Stability}

System (7) defines a three-dimensional discrete-time system (map) of the form

$$
\tilde{z}=A z, \quad z, \tilde{z} \in \mathbb{R}^{3}, A \in \mathbb{R}^{3 \times 3}
$$

with fixed point $z_{0}=(0,0,0)^{T}$. The stability of the above system is governed by the characteristic multipliers $\mu$ (eigenvalues of matrix $A$ ) and there are three ways in which instability may occur: either a positive or a negative multiplier crosses the unit circle $\left(\mu_{1}= \pm 1\right)$ or a pair of complex multipliers cross the unit circle and we have $\mu_{1,2}=e^{ \pm i \theta}$ for some $0<\theta<\pi$. For further details, see e.g. [7].

Straightforward calculation reveals that the eigenvalues are

$$
\lambda_{1,2}=\frac{1}{2}\left(1+\alpha \pm \sqrt{(1-\alpha)^{2}+8 \beta}\right) \text { and } \lambda_{3}=0 .
$$

The zero eigenvalue corresponds to the second equation in (7). We have a flip (period-doubling) bifurcation if $\mu=-1$, which occurs at

$$
P_{\text {flip }}=2 \frac{A_{p}}{K} f \text {. }
$$

Beyond this value, a period two cycle appears, with period $2 \Delta t$. On the other hand, we have a Neimark-Sacker bifurcation if the eigenvalues are complex numbers with unit magnitude, which happens at

$$
P_{N S}=\frac{I}{2 f},
$$

and the frequency of the appearing oscillation depends on the phase angle of the eigenvalues, i.e. $\theta / 2 / \pi$.

Note that the location of the flip bifurcation is independent of the integrator term, while the Neimark-Sacker point linearly increases with the integrator term. For a given integrator gain and sampling frequency, the stable region is where $P_{N S}<P<P_{\text {flip }}$ and this region shrinks if the sampling frequency is decreased, as depicted in Figure 3 . Moreover, these curves join at

$$
f_{\text {crit }}=\frac{1}{2} \sqrt{\frac{K I}{A_{p}}}, \quad \text { and } \quad P_{c r i t}=\sqrt{\frac{A_{p} I}{K}},
$$

i.e. for $f<f_{\text {crit }}$, no stable motion is possible and the control is unconditionally unstable.

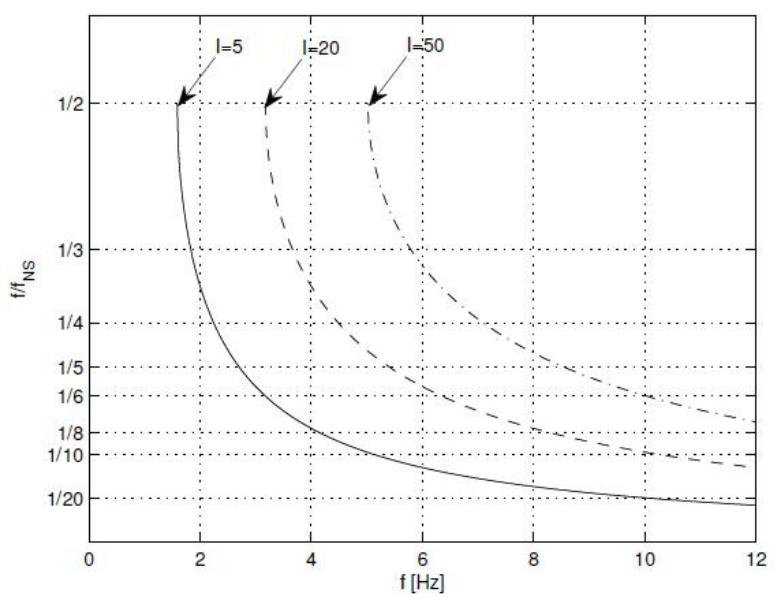

Fig. 6. The frequency of the appearing oscillation on the Neimark-Sacker border, for $I=5$ (solid line), $I=20$ (dashed line) and $I=50$ (dash-dot line).

The temporal behaviour of the system is depicted on Fig. 4. The corresponding numerical values were $I=20, f=10$ and $P=[0.6,1.5,9,10.1]$. The first simulation (left-upper corner) at $P=0.6$ is just beneath the Neimark-Sacker stability limit and a low-frequency unstable oscillation can be seen. The next two simulations (right upper and left bottom) are at $P=1.5$ and $P=9$, respectively, both ones in the stable region, however, the previous one close to the Neimark-Sacker border while the latter one close to the flip boundary. The last simulation (right bottom) was obtained with $P=10.1$, which is already above the flip limit and, indeed, the frequency of the limit cycle is twice the sampling frequency.

\subsection{Comparison with measurements}

We present the comparison between the experiments and the theory in Fig. 5. which depicts the critical $P$ values as a function of the sampling frequency for several integrator gains. Note that during the experiments, those parameter values were marked as 'unstable', for which the relative valve displacement became larger than $75 \%$ of the full stroke.

The theory suggests that the upper stability limit (corresponding to flip bifurcation) does not depend on the integrator gain, see (11). However, the measurements show that it slightly decreases with increasing integrator gain. The Neimark-Sacker limit (lower bound) obtained experimentally changes with the integrator gain in accordance to the theory, i.e. (12).

Fig. 6 depicts the frequency of the appearing oscillation on the Neimark-Sacker line for several integrator gains. All curves terminate at $1 / 2$ at $f_{\text {crit }}$ - given by (13) - where the NS and the flip curves join. Note that the higher the sampling frequency is, the lower the relative oscillation frequency $\left(f / f_{N S}\right)$ becomes. The ratio of the two frequencies can be as low as $1 / 20$. At the same time, an increase in the integrator gain decreases the oscillation frequency as well. 


\section{Summary}

We presented a series of measurements on the effect of sample time delay on the stability of the PI position control of a hydraulic cylinder. A simplified flow control valve model allowed the construction of a simple, linear three-dimensional map, whose stability can be analysed by standard techniques. These computations revealed that there are two mechanisms of loosing stability. The first one is a flip bifurcation, were the period of the appearing limit cycle is twice the period of the sampling rate. This stability border increases linearly with the frequency and is independent of the integrator gain. The other instability mechanism is a Neimark-Sacker bifurcation, which is present for low proportional gains and gives birth of lowfrequency oscillations. Moreover, the relative frequency $f / f_{N S}$ of the oscillations further decreases with increasing the sampling rate frequency but slightly increases with increasing integrator gains. It was shown that there exists a critical sampling rate, below which no stable control exists. It is worth mentioning that these are exactly the same kind of mechanisms that arise in the stability analysis of high speed milling, see [9].

We have found a good accordance with the experimental results, which is somewhat surprising given the simplicity of the mathematical model. However, it shall be emphasised that we analysed a low-load case, i.e. the cylinder is 'oversized' in the sense that the dynamics of the moved object can be neglected compared to the piston force.

\section{References}

1 Chen J, Dixon W, Wagner J, Dawson DM, Exponential TRacking Control of a Hydraulic Proportional Directional Valve and Cylinder via Integrator Backstepping, In: Proceedings of IMECE 02, 2002 ASME International Mechanical Engineering Congress and Expo, Vol. IMECE2002-32076, 2002. paper No. IMECE2002-32076.

2 Hong M, Lin C, Shiu B, Stabilizing network control for pneumatic systems with time-delays, Mechatronics, 19(3), (2009), 399-409, DOI 10.1016/j.mechatronics.2008.09.008

3 Li S, Tao B, Peng S, Zimming W, Sampled-data control of networked linear control systems, Automatica, 43(5), (2007), 903-911, DOI 10.1016/j.automatica.2006.11.015

4 Insperger T, Stépán G, Act-and-wait control concept for discrete-time systems with feedback delay, IET Control Theory and Applications, 1(3), (2007), 553-557, DOI 10.1049/iet-cts:20060051

5 Jelali M, Kroll A, Hydraulic Servo-systems: modelling, identification and control, Springer, 2004.

6 Kuznetsov Y, Elements of Applied Bifurcation Theory, In: Elements of Applied Bifurcation Theory, Applied Mathematical Sciences, Vol. 112, Springer, 2004, DOI 10.1007/978-1-4757-3978-7

7 Liu Y, Handroos $\mathbf{H}$, Sliding mode control for a class of hydraulic position servo, Mechatronics, 9(1), (1999), 111-123, DOI 10.1016/S09574158(98)00044-0

8 Stépán G, Vibrations of machines subjected to digital force control, International Journal of Solids and Structures, 38(10-13), (2001), 2149-2159, DOI 10.1016/S0020-7683(00)00158-X

9 Stepan G, Hogan S, Szalai R, Global dynamics of low immersion highspeed milling, Chaos, 14(4), (2004), 1069-1077, DOI 10.1063/1.1807395

$10 \mathbf{N}$ van de Wouw, Nei D, Heemels W, A discrete-time framework for stability analysis of nonlinear networked control systems, Automatica, 48(6), (2012), 1144-1153, DOI 10.1016/j.automatica.2012.03.005

11 Wang Y-J, Characterization and quenching of friction-induced limit cycles of electro-hydraulic servovalve control systems with transport delay, ISA Transactions, 49(4), (2010), 489-500, DOI 10.1016/j.isatra.2010.05.002 